IdeAs

Idées d'Amériques

9 | 2017

Poètes et éditeurs : diffuser la poésie d'avant-garde américaine (depuis 1945)

\title{
Guerre des images et Hollywood patriote
}

\section{Clémentine Tholas-Disset}

\section{(2) OpenEdition \\ Journals}

Édition électronique

URL : https://journals.openedition.org/ideas/1865

DOI : $10.4000 /$ ideas. 1865

ISSN : 1950-5701

Éditeur

Institut des Amériques

Référence électronique

Clémentine Tholas-Disset, " Guerre des images et Hollywood patriote », IdeAs [En ligne], 9 | 2017, mis en ligne le 12 juillet 2017, consulté le 20 octobre 2022. URL : http://journals.openedition.org/ideas/

1865 ; DOI : https://doi.org/10.4000/ideas. 1865

Ce document a été généré automatiquement le 20 octobre 2022

\section{(c) (i) ()}

Creative Commons - Attribution - Pas d'Utilisation Commerciale - Pas de Modification 4.0 International - CC BY-NC-ND 4.0

https://creativecommons.org/licenses/by-nc-nd/4.0/ 


\title{
Guerre des images et Hollywood patriote
}

\author{
Clémentine Tholas-Disset
}

1 Si depuis près d'un siècle, la mémoire de la Première Guerre mondiale est largement influencée par les œuvres cinématographiques internationales telles que Les Quatre cavaliers de l'Apocalypse (1921), À l'Ouest, rien de nouveau (1930), La Grande illusion (1937), Les Sentiers de la gloire (1957), La Chambre des officiers (2001), Un Long dimanche de fiançailles (2004), Cheval de guerre (2011), c'est parce que, durant la Grande Guerre, le cinéma est devenu un outil de communication et de persuasion indispensable pour les pays belligérants. Le cinéma américain s'est alors imposé comme modèle culturel pour le reste du monde.

2 Lors de l'entrée en guerre des États-Unis, le président Woodrow Wilson réquisitionne la nation tout entière et de nombreux services pour appuyer l'effort de guerre. Le cinéma est mobilisé pour justifier l'engagement américain dans le conflit et convertir le monde aux valeurs américaines. Sur ordre de Wilson, le 13 avril 1917, le Committee on Public Information (CPI) est créé pour diriger l'information et la communication de guerre auprès de la population américaine et des autres pays. À la tête de ce comité de propagande, l'ancien journaliste George Creel considère que la conquête des esprits est un véritable deuxième front. Le CPI met tout en œuvre pour convaincre le pays du bienfondé de la guerre et de la mission des États-Unis en tant que guide pour les autres nations, se faisant la voix de la diplomatie morale prônée par le président. Reconnaissant le pouvoir du cinéma comme média de masse international, Wilson et Creel dotent le CPI d'une division spécialisée dans les films. Cette antenne réalise et distribue de longs-métrages documentaires sur la puissance militaire américaine ${ }^{1}$, montés à partir d'images filmées par les transmissions (US signal corps), ainsi que des actualités hebdomadaires - intitulées The Official War Review - également élaborées avec les archives visuelles militaires. Ces productions officielles n'ont pas le succès escompté. Elles regroupent de manière assez disparate des images tournées au hasard par des opérateurs militaires et les distributeurs ne souhaitent pas placer en salle des films de propagande gouvernementale qui, selon eux, n'attireront pas de spectateurs. 
Vexé par cette réaction de la profession cinématographique, Creel demande à la CroixRouge de diffuser ses films et les met aussi à disposition d'autres associations. Craignant la concurrence déloyale de films diffusés gratuitement dans d'autres réseaux, Hollywood accepte un compromis: l'abandon de la distribution parallèle contre l'acceptation de mettre dans chaque cargaison de films de divertissement pour le marché étranger $20 \%$ de films éducatifs émanant du CPI. Les exploitants européens ne peuvent obtenir de films hollywoodiens que s'ils s'engagent à diffuser les productions du gouvernement américain et les licences d'exportation des films à l'étranger sont délivrés par le comité Creel. De plus, les exploitants de salle américains acceptent aussi de collaborer en accueillant les Four-minute men, soixante-quinze mille bénévoles mandatés par le CPI pour faire de courts discours sur l'engagement du pays pendant les changements de bobine entre deux films; et les cinémas se transforment ainsi en terrain de propagande.

3 En 1917, l'industrie américaine du film comprend qu'elle peut prospérer si elle fait coïncider ses intérêts avec ceux de Washington et s'engage dans la voie d'un patriotisme commode (practical patriotism) en soutenant les stratégies du CPI et le message du président. Cette industrie culturelle est gérée par la National Association of the Motion Picture Industry (NAMPI), créée en 1916 pour coordonner les relations entre tous les secteurs de la profession - acteurs, producteurs, ingénieurs, distributeurs, exploitants de salle, publicitaires. Syndicat du cinéma, la NAMPI est chapeautée par le producteur William Brady. L'association veille aux relations entre Hollywood et le gouvernement américain, en particulier concernant les problèmes légaux et les échanges commerciaux internationaux. Pour compléter son accord commercial avec Washington concernant les exportations, l'association s'engage dans différentes initiatives de soutien à la guerre, en formant en mai 1917 un comité de coopération à la guerre et en participant au ravitaillement et à la promotion des emprunts de guerre ou « emprunts de la liberté » (liberty loans). Le 28 juin 1917, le président Wilson adresse une lettre à Brady lui expliquant que le langage universel du cinéma doit servir à mettre en valeur les projets américains et il reconnaît le dévouement des professionnels du cinéma à la cause patriotique. C'est une victoire pour Brady qui souhaite que le cinéma soit considéré comme une industrie vitale pour l'effort de guerre. En effet, il craint de voir les salles de cinéma se vider si le gouvernement présente le cinéma comme une activité non patriotique qui encourage les citoyens à s'amuser et à dépenser en frivolités alors que l'heure est à la gravité et au rationnement. De plus, alors que certains hommes politiques comme Théodore Roosevelt fustigent le manque de loyauté des "Américains à trait d'union" (hyphenated Americans) qui n'abandonnent pas leur identité d'origine, l'industrie du film est dans une position délicate: plusieurs producteurs sont originaires d'Allemagne ${ }^{2}$ et craignent d'être accusés de double allégeance, voire de collusion avec l'ennemi.

Le soutien de l'industrie cinématographique à la guerre s'articule autour du contenu des films et des actions menées par les stars. À la demande du secrétaire au Trésor William MacAdoo, les têtes d'affiche, réunies au sein de la Motion Picture War Service Association, apportent leur concours à l'US Food Administration (USFA), aux campagnes pour enjoindre la population de souscrire aux «emprunts de la liberté » et lèvent régulièrement des fonds pour la Croix-Rouge. Ainsi, de Mabel Normand à Dorothy Gish, en passant par les emblématiques Mary Pickford, Charles Chaplin et Douglas Fairbanks, les acteurs s'adressent au public dans des films ou pendant les Liberty Loan Drives organisées à travers tout le pays pendant lesquelles ils galvanisent les foules endiablées 
avec des discours patriotiques. En 1918, lors de la quatrième campagne des « emprunts de la liberté ", les vedettes tournent également des courts-métrages publicitaires incitant à continuer les dons, parmi lesquels The Bond: A Liberty Loan Appeal de Chaplin et One Hundred Percent American avec Pickford qui ont été préservés. La jeune actrice est également nommée marraine du $143^{\mathrm{e}}$ bataillon d'artillerie de Californie et de plusieurs pilotes à qui elle rend visite pendant leur entraînement. D'autres professionnels du film manifestent leur fibre patriotique en se tenant prêts à agir pour défendre le pays, comme le cinéaste Cecil B. DeMille qui coordonne la Lasky Home Guard, une milice de volontaires du studio Lasky-Famous Players. DeMille et Jessy Lasky sont persuadés qu'il faut aider les hommes à se préparer au cas où ils seraient appelés lors d'une nouvelle vague de conscription et ils leur proposent un entraînement militaire rudimentaire avant qu'ils ne rejoignent l'armée. Quant à Buster Keaton, il part sous les drapeaux en 1917, dans la 40 e division d'infanterie, mais suite à une infection de l'oreille, il sert en France en tant que cryptographe. À la fin de la guerre, il forme avec d'autres soldats de son unité la troupe théâtrale des Sunshine Players pour divertir les soldats américains grâce à des performances burlesques et des numéros de travesti. Moins conventionnelle, cette contribution n'en est pas moins importante puisqu'elle permet de regonfler le moral de troupes épuisées au moment de la démobilisation.

5 Les films de guerre tournés par Hollywood incitent la population à soutenir la participation du pays au conflit. Il faut toutefois noter que les films ayant directement trait à la guerre ne représentent qu'une minorité parmi les films produits pendant les dix-neuf mois de mobilisation américaine. En avril 1917, 14\% des films ont pour thème la guerre en cours contre $23 \%$ en octobre 1918. Une partie de l'industrie cinématographique est persuadée qu'il est impératif d'offrir aux spectateurs des distractions qui leur permettent de s'évader tandis que certains professionnels pensent, au contraire, que les films doivent illustrer l'actualité. Plusieurs studios affirment qu'en temps de guerre le cinéma a l'obligation de véhiculer de la gaîté et qu'il faudrait éliminer des catalogues les œuvres contenant des éléments déprimants ou tragiques. Les films de guerre constituent ainsi une faible proportion dans l'ensemble des films distribués pendant le conflit; les producteurs les mettent néanmoins en avant comme des films de prestige dont la sortie s'accompagne d'opérations promotionnelles coûteuses et spectaculaires dans des salles luxueuses. L'Amérique ne subissant pas le conflit sur son sol, Hollywood peut continuer de tourner ses films et d'approvisionner le marché américain et international. Ses films s'imposent comme un des divertissements majeurs des différents pays d'Europe qui peinent à faire fonctionner leur industrie cinématographique locale à cause des restrictions dues à la guerre et aux combats. Même en Allemagne, où se met pourtant en place dès 1917 une riposte cinématographique avec la BUFA (Bild- und Filmamt) qui assure la circulation de l'information, certains films hollywoodiens remportent de grands succès, en particulier ceux de Chaplin. Avant l'automne 1917, le contenu des productions hollywoodiennes concernant la guerre se veut relativement neutre pour satisfaire un marché intérieur complexe qui réunit à la fois des pacifistes, des sympathisants de l'Allemagne, notamment dans le Midwest où la population d'origine germanique est importante, des partisans de la Grande-Bretagne et des militants pour la préparation au combat. De plus, il est important que les films soient adaptés au goût et au positionnement idéologique du marché étranger, notamment britannique, puisque les Britanniques s'avèrent être les plus grands consommateurs de films américains pendant le conflit. Pour ces raisons commerciales, Hollywood propose des œuvres valorisant davantage les 
membres de la Triple-Entente (Grande-Bretagne, France, Russie) que l'Allemagne. Par exemple, The Spirit of '76, sur la guerre d'Indépendance, est d'abord censuré par de nombreuses coupes après sa sortie en mai 1917 puis retiré des écrans car accusé d'attaquer les alliés anglais et de soutenir l'Allemagne. Son producteur, Robert Goldstein, d'origine allemande lointaine, est condamné à dix ans de prison pour violation de l'Espionage Act. Même si les films doivent alors mettre en valeur la neutralité des États-Unis, leur contenu est fortement orienté.

Les films dont l'intrigue est liée à la guerre sont généralement des mélodrames obéissant à une vision parfois manichéenne du conflit et célébrant l'héroïsme de jeunes, engagés comme soldats ou infirmières, qui défendent avec courage les idéaux démocratiques des États-Unis. Si, avant 1917, Hollywood affiche des velléités pacifistes en accord avec le rejet d'une guerre qui ne concerne pas les Américains, on observe ensuite un revirement férocement patriotique qui se traduit par des films " anti-Huns " (hate-the-hun films). À l'instar de The Little American réalisé par Cecil B. DeMille et avec Mary Pickord, ces productions dépeignent les Allemands comme des brutes barbares, capables des pires atrocités. Elles renforcent l'antagonisme vis-à-vis des Allemands d'Europe et des Allemands des États-Unis, considérés comme des traitres dont on doit boycotter les journaux et les églises luthériennes. Ces films jouent un rôle notoire dans le dénigrement de la culture allemande et l'exacerbation de la peur des espions sur le sol américain. Ils encouragent un "super-patriotisme » américain, selon le terme proposé par Leslie Midkiff Debauche ${ }^{3}$, construit autour de la haine du Teuton et amènent les Américains à attaquer certains de leurs compatriotes du fait de leur patronyme ou de leur origine, comme Robert Paul Prager lynché par les habitants de Collinsville (Illinois) où il s'est établi treize ans plus tôt. À l'hystérie anti-germanique vient s'ajouter, dans d'autres films, le rejet virulent du pacifisme ou la critique acerbe des hommes qui ne combattent pas, considérés comme des lâches qu'il faut rabaisser. Toutefois, les comédies de guerre proposées par les vedettes les plus patriotes, telles que Chaplin et Pickford, viennent contrebalancer le message agressif ou désespéré des mélodrames en se riant du Kaiser Guillaume II, du réveil patriotique inattendu des États-Unis, du décalage entre les préoccupations de la population et celles des gouvernements, et surtout du non-sens de la guerre en général. Dans les films burlesques comme La Petite vivandière (Johanna Enlists) ou Charlot soldat (Shoulder Arms), le conflit mondial est dédramatisé, la violence du massacre éludée, et la guerre donnée à voir aux Américains comme un jeu d'enfants dans lequel la victoire sera facile. Pour rassurer, les comédies altèrent la réalité du conflit au point de la faire oublier, tout en saisissant certains aspects cruciaux comme le ressenti des soldats ou des populations civiles. Par exemple, même si certains reprochent à Chaplin de ne pas se battre, Charlot soldat est fortement plébiscité par les soldats qui considèrent le film comme une interprétation fidèle de leur quotidien au front. La dimension humaniste du film séduit autant que ses gags, les soldats disant retrouver leurs mésaventures dans les péripéties de Charlot.

Quand les États-Unis interviennent en 1917, le pays et son cinéma se lancent dans une guerre des images pour rallier la population américaine à l'effort national mais aussi pour vendre l'Amérique au monde. Les films hollywoodiens tournés alors se répandent sur le marché étranger sans susciter de concurrence réelle à cette colonisation culturelle, sauf peut-être en Allemagne. De plus, une partie de ces œuvres met en scène 
les Américains comme des sauveurs et des champions de la démocratie, offrant un nouveau support à l'ancestral exceptionnalisme états-unien.

\section{NOTES}

1. Les longs-métrages documentaires du CPI sont: Pershing's Crusaders, America's Answer, Under Four Flags

2. Par exemple, Carl Laemmle le dirigeant d'Universal et Harry Cohn de Columbia.

3. Leslie Midkiff Debauche, Reel Patriotism: The Movies and World War I, Madison, University of Wisconsin, 1997.

\section{AUTEUR}

\section{CLÉMENTINE THOLAS-DISSET}

Clémentine Tholas-Disset est maître de conférences en civilisation américaine à l'université Paris III-Sorbonne Nouvelle. Spécialiste du cinéma hollywoodien et de la propagande de la Première Guerre mondiale, elle est l'auteur de Le Cinéma muet américain et ses premiers récits filmiques (2014) et a co-dirigé l'ouvrage collectif Humor, Entertainment and Popular Culture during World War One (2015). 